\section{Journal of Sleep Research}

Helga Peter ${ }^{1}$ und Thomas Penzel ${ }^{2}$

${ }^{1}$ Marburg, Deutschland

${ }^{2}$ Interdisziplinäres Schlafmedizinisches Zentrum, Charité -

Universitätsmedizin Berlin, Berlin, Deutschland

\section{Definition}

Publikationsorgan der European Sleep Research Society. Siehe auch $\triangleright$,Schlafmedizinische Fachgesellschaften, Fachzeitschriften und Publikationsforen“. 